\title{
Cauchy problem for some fourth-order nonstrictly hyperbolic equations
}

\author{
V. I. Korzyuk, N. V. Vinh \\ Institute of Mathematics, Belarusain Academy of Sciences, Belarusian State University, Belarus \\ korzyuk@bsu.by,vinhnguyen0109@gmail.com
}

\section{DOI 10.17586/2220-8054-2016-7-5-869-879}

\begin{abstract}
We describe the analytic solution of the Cauchy problem for some fourth-order linear hyperbolic equations with constant coefficients in a halfplane in the case of two independent variables, assuming certain conditions for the coefficients. Suitable conditions are assumed for the coefficients, and the equation operator is composed of first-order linear operators.
\end{abstract}

Keywords: Cauchy problem, analytic solution, fourth-order hyperbolic equations, nonstrictly hyperbolic equations.

Received: 18 April 2016

Revised: 5 May 2016

\section{Introduction}

This work is devoted to considering the Cauchy problem on a half-plane for some fourth-order nonstrictly hyperbolic linear equation with constant coefficients. The operator appearing in the equation involves a composition of first-order differential operators. The Cauchy problem for such an equation was previously considered in [1,2] in the case of a strictly hyperbolic equation (a Petrovskii hyperbolic equation [3,4]). The general solution for both strictly and nonstrictly hyperbolic equations of arbitrary order was constructed there as well. The case of a nonstrictly hyperbolic equation with the coincidence of all characteristics was considered in [5], and the solutions of the Cauchy problem in all cases of a nonstrictly hyperbolic third-order equation of such a form were obtained in [6].

Unique solvability of the problem and the construction of solution for the Cauchy problem is one of the classical problems in the theory of differential equations. Differential equations arise in the modelling of several natural phenomena, and the Cauchy problem is one of the first and most important.

The Cauchy problem for hyperbolic partial differential equations was studied by many mathematicians for a long time, mostly by methods of functional analysis. In this paper, we suggest the following analytical methods for solving the Cauchy problem. First, we find the general solutions using the characteristics of the equation. Next, from the general solution, we determine the solution which satisfies the Cauchy conditions. The latter step is the main difficulty for the determination of the required analytical solution. The general solution of the homogeneous equation depends on a number of arbitrary functions. To determine them, we use Cauchy differential conditions. This leads to the corresponding system of differential equations. In each particular case, solving this system requires a different method and approach. In addition, the solutions depend on a number of arbitrary constants. In order to prove the uniqueness of the solution for a given Cauchy problem, it is necessary to prove that all these arbitrary constants are cross-eliminated after substitution into the general solution.

Partial differential equations of fourth order are encountered when studying mathematical models for certain natural and physical processes. An example of such type of equations, is the fourth-order governing differential equation for nanorod based on nonlocal second-order strain gradient model [see [7,8]]:

$$
E A\left(\epsilon_{0} a\right)^{2} \frac{\partial^{4} u(t, x)}{\partial x^{4}}+E A \frac{\partial^{2} u(t, x)}{\partial x^{2}}-\rho A \frac{\partial^{2} u(t, x)}{\partial t^{2}}=0,
$$

and the flexural wave equation for an Euler-Bernoulli beam has a fourth order derivative in space and is given as [see $[9,10]]$ :

$$
E I \frac{\partial^{4} u(t, x)}{\partial x^{4}}+\eta A \frac{\partial u(t, x)}{\partial t}+\rho A \frac{\partial^{2} u(t, x)}{\partial t^{2}}=0 .
$$

On the plane $\mathbb{R}^{2}$ of two independent variables $t$ and $x$, we introduce the half-plane $Q=[0,+\infty) \times \mathbb{R}$ on which we consider the following partial differential equation of fourth-order, for a function $u: \mathbb{R}^{2} \ni Q \supset(t, x) \rightarrow$ $u(t, x) \in \mathbb{R}:$

$$
\mathcal{L}^{(4)} u(t, x)=\prod_{k=1}^{4}\left(\partial_{t}-a^{(k)} \partial_{x}+b^{(k)}\right) u(t, x)=f(t, x), \quad(t, x) \in Q
$$


together with the initial conditions:

$$
\left.u\right|_{t=0}=\varphi_{0}(x),\left.\quad \frac{\partial u}{\partial t}\right|_{t=0}=\varphi_{1}(x),\left.\quad \frac{\partial^{2} u}{\partial t^{2}}\right|_{t=0}=\varphi_{2}(x),\left.\quad \frac{\partial^{3} u}{\partial t^{3}}\right|_{t=0}=\varphi_{3}(x),
$$

where $\partial_{t}=\frac{\partial}{\partial t}, \partial_{x}=\frac{\partial}{\partial x}$ - are the first derivatives with respect to $t, x$ and $a^{(k)}, b^{(k)}$ are given real numbers, $f: \bar{Q} \rightarrow \mathbb{R}$ with $\bar{Q}=[0,+\infty) \times \mathbb{R}$, the closure of $Q$.

Consider the fourth-order homogeneous equation:

$$
\mathcal{L}^{(4)} u(t, x)=0, \quad(t, x) \in Q,
$$

with

$$
\mathcal{L}^{(4)}=\prod_{k=1}^{p}\left(\partial_{t}-a^{(k)} \partial_{x}+b^{(k)}\right)^{r(k)},
$$

where $p$ and $r^{(k)}$ are positive integers such that $p \leq 4$ and $r^{(1)}+r^{(2)}+\ldots+r^{(p)}=4$. By [2], the general solution of Eq. (5) has the form:

$$
u(t, x)=\sum_{k=1}^{p} e^{-b^{(k)}} t \sum_{s=1}^{r(k)} t^{s-1} f^{(k s)}\left(x+a^{(k)} t\right) .
$$

In this paper, we study five cases of fourth-order non-strictly hyperbolic equations, in particular:

Case 1: $r^{(1)}=4$ and $r^{(j)}=0, j=\overline{2,4}$.

Case 2: $r^{(1)}=3, r^{(2)}=1$ and $r^{(m)}=0$ with $m=3,4$.

Case 3: $r^{(1)}=2, r^{(2)}=2$ and $r(m)=0$ with $m=3,4$.

Case 4: $r^{(1)}=2, r^{(2)}=1, r^{(3)}=1$ and $r^{(4)}=0$.

Case 5: $r^{(i)}=1 \forall i=\overline{1,4}$.

\section{Main results}

In this section, we consider the some cases for fourth-order non-strictly hyperbolic equations of the form (5).

Case 1: $r^{(1)}=4$ and $r^{(j)}=0, j=\overline{2,4}$. Assume that the coefficients of (5) satisfy $a^{(k)}=a, b^{(k)}=b, \forall k=\overline{1,4}$. Then, according to (7), the general solution of (5) can be written in the following form:

$$
u(t, x)=e^{-b t}\left(f_{1}(x+a t)+t f_{2}(x+a t)+t^{2} f_{3}(x+a t)+t^{3} f_{4}(x+a t)\right) .
$$

By plugging (8) into (4), after simplifying, we obtain:

$$
\begin{aligned}
f_{1}(x) & =\varphi_{0}(x) ; \\
-b f_{1}(x)+f_{2}(x)+a f_{1}^{\prime}(x) & =\varphi_{1}(x) ; \\
b^{2} f_{1}(x)+2 f_{3}(x)-2 b\left(f_{2}(x)+a f_{1}^{\prime}(x)\right)+2 a f_{2}^{\prime}(x)+a^{2} f_{1}^{\prime \prime}(x) & =\varphi_{2}(x) ; \\
-b^{3} f_{1}(x)+6 f_{4}(x)+3 b^{2}\left(f_{2}(x)+a f_{1}^{\prime}(x)\right)+6 a f_{3}^{\prime}(x) & \\
-3 b\left(2 f_{3}(x)+2 a f_{2}^{\prime}(x)+a^{2} f_{1}^{\prime \prime}(x)\right)+3 a^{2} f_{2}^{\prime \prime}(x)+a^{3} f_{1}^{\prime \prime \prime}(x) & =\varphi_{3}(x) ;
\end{aligned}
$$

or equivalently:

$$
\begin{aligned}
f_{1}(x) & =\varphi_{0}(x) \\
f_{2}(x) & =\varphi_{1}(x)+b \varphi_{0}(x)-a \varphi_{0}^{\prime}(x) ; \\
f_{3}(x) & =\frac{1}{2}\left(\varphi_{2}(x)+b^{2} \varphi_{0}(x)+2 b \varphi_{1}(x)-2 a \varphi_{1}^{\prime}(x)-2 a b \varphi_{0}^{\prime}(x)+a^{2} \varphi_{0}^{\prime \prime}(x)\right) ; \\
f_{4}(x) & =\frac{1}{6}\left(\varphi_{3}(x)+b^{3} \varphi_{0}(x)-3 b^{2}\left(\varphi_{1}(x)+b \varphi_{0}(x)\right)\right. \\
& -3 a\left(\varphi_{2}^{\prime}(x)+2 b \varphi_{1}^{\prime}(x)+b^{2} \varphi_{0}^{\prime}(x)-2 a \varphi_{1}^{\prime \prime}(x)-2 a b \varphi_{0}^{\prime \prime}(x)+a^{2} \varphi_{0}^{\prime \prime \prime}(x)\right) \\
& \left.+3 b\left(\varphi_{2}(x)+b^{2} \varphi_{0}(x)+2 b \varphi_{1}(x)\right)-3 a^{2}\left(\varphi_{1}^{\prime \prime}(x)+b \varphi_{0}^{\prime \prime}(x)-a \varphi_{0}^{\prime \prime \prime}(x)\right)-a^{3} \varphi_{0}^{\prime \prime \prime}(x)\right) .
\end{aligned}
$$


Substituting $f_{1}(x), f_{2}(x), f_{3}(x), f_{4}(x)$ into (8), finally we have:

$$
\begin{aligned}
& u(t, x)=\frac{1}{6} e^{-b t}\left(\left(6+6 b t+3 b^{2} t^{2}+b^{3} t^{3}\right) \varphi_{0}(x+a t)+t\left(3\left(2+2 b t+b^{2} t^{2}\right) \varphi_{1}(x+a t)\right.\right. \\
& +3 t(1+b t) \varphi_{2}(x+a t)+t^{2} \varphi_{3}(x+a t)-6 a \varphi_{0}^{\prime}(x+a t)-6 a b t \varphi_{0}^{\prime}(x+a t)-3 a b^{2} t^{2} \varphi_{0}^{\prime}(x+a t) \\
& -6 a t \varphi_{1}^{\prime}(x+a t)-6 a b t^{2} \varphi_{1}^{\prime}(x+a t)-3 a t^{2} \varphi_{2}^{\prime}(x+a t)+3 a^{2} t \varphi_{0}^{\prime \prime}(x+a t) \\
& \left.+3 a^{2} b t^{2} \varphi_{0}^{\prime \prime}(x+a t)+3 a^{2} t^{2} \varphi_{1}^{\prime \prime}(x+a t)-a^{3} t^{2} \varphi_{0}^{\prime \prime \prime}(x+a t)\right) .
\end{aligned}
$$

Case 2: The coefficients of (5) satisfy $a^{(k)}=a, b^{(k)}=b$ with $k=1,2,3$ and $a^{(4)} \neq a, b^{(k)}$ - arbitrary constants. According to (7), the general solution of equation (5) has the form:

$$
u(t, x)=e^{-b t}\left(f_{1}(x+a t)+t f_{2}(x+a t)+t^{2} f_{3}(x+a t)\right)+e^{-b^{(4)}} f_{4}\left(x+a^{(4)} t\right) .
$$

Similarly, due to (4), we get the following system of differential equations for the functions $f_{k}(x)$ with $k=1,2,3,4$ :

$$
\begin{array}{r}
f_{1}(x)+f_{4}(x)=\varphi_{0}(x) ; \\
-b f_{1}(x)+f_{2}(x)-b^{(4)} f_{4}(x)+a f_{1}^{\prime}(x)+a^{(4)} f_{4}^{\prime}(x)=\varphi_{1}(x) ; \\
b^{2} f_{1}(x)+2 f_{3}(x)+\left(b^{(4)}\right)^{2} f_{4}(x)-2 b\left(f_{2}(x)+a f_{1}^{\prime}(x)\right)+2 a f_{2}^{\prime}(x)-2 a^{(4)} b^{(4)} f_{4}^{\prime}(x) \\
+a^{2} f_{1}^{\prime \prime}(x)+\left(a^{(4)}\right)^{2} f_{4}^{\prime \prime}(x)=\varphi_{2}(x) ; \\
-b^{3} f_{1}(x)-\left(b^{(4)}\right)^{3} f_{4}(x)+3 b^{2}\left(f_{2}(x)+a f_{1}^{\prime}(x)\right)+6 a f_{3}^{\prime}(x)+3 a^{(4)}\left(b^{(4)}\right)^{2} f_{4}^{\prime}(x)+3 a^{2} f_{2}^{\prime \prime}(x) \\
\left.-3 b\left(2 f_{3}(x)\right)+2 a f_{2}^{\prime}(x)+a^{2} f_{1}^{\prime \prime}(x)\right)-3 b^{(4)}\left(a^{(4)}\right)^{2} f_{4}^{\prime \prime}(x)+a^{3} f_{1}^{\prime \prime \prime}(x)+\left(a^{(4)}\right)^{3} f_{4}^{\prime \prime \prime}(x)=\varphi_{3}(x) .
\end{array}
$$

For the sake of convenience, we introduce the following notations of differential operators: $d_{1}=a d / d x-b$, $d_{4}=a^{(4)} d / d x-b^{(4)}$ and $d_{1}^{j}=(a d / d x-b)^{j}, d_{4}^{j}=\left(a^{(4)} d / d x-b^{(4)}\right)^{j}$. The system of differential equations for the unknown function $f_{1}(x), f_{2}(x), f_{3}(x), f_{4}(x)$ becomes:

$$
\begin{aligned}
f_{1}(x)+f_{4}(x) & =\varphi_{0}(x) ; \\
d_{1} f_{1}(x)+f_{2}(x)+d_{4} f_{4}(x) & =\varphi_{1}(x) ; \\
d_{1}^{2} f_{1}(x)+2 d_{1} f_{2}(x)+2 f_{3}(x)+d_{4}^{2} f_{4}(x) & =\varphi_{2}(x) ; \\
d_{1}^{3} f_{1}(x)+3 d_{1}^{2} f_{2}(x)+6 d_{1} f_{3}(x)+d_{4}^{3} f_{4}(x) & =\varphi_{3}(x) .
\end{aligned}
$$

Observe that the preceding system can be reduced to the one of differential equations with diagonal matrix. To this end, we apply the operator $d_{4}$ to the first three equations in the system and subtract every other equation of the resulting system from the preceding one. As a result, we obtain:

$$
\begin{aligned}
f_{1}(x)+f_{4}(x) & =\varphi_{0}(x) ; \\
\left(d_{1}-d_{4}\right) f_{1}(x)+f_{2}(x) & =\varphi_{1}(x)-d_{4} \varphi_{0}(x) ; \\
\left(d_{1}^{2}-d_{1} d_{4}\right) f_{1}(x)+\left(2 d_{1}-d_{4}\right) f_{2}(x)+2 f_{3}(x) & =\varphi_{2}(x)-d_{4} \varphi_{1}(x) ; \\
\left(d_{1}^{3}-d_{1}^{2} d_{4}\right) f_{1}(x)+\left(3 d_{1}^{2}-2 d_{1} d_{4}\right) f_{2}(x)+\left(6 d_{1}-2 d_{4}\right) f_{3}(x) & =\varphi_{3}(x)-d_{4} \varphi_{2}(x) .
\end{aligned}
$$

By continuing transformations in a similar way, we receive:

$$
\begin{aligned}
f_{1}(x)+f_{4}(x) & =\varphi_{0}(x) ; \\
\left(d_{1}-d_{4}\right) f_{1}(x)+f_{2}(x) & =\varphi_{1}(x)-d_{4} \varphi_{0}(x) ; \\
\left(d_{1}^{2}-d_{1} d_{4}\right) f_{1}(x)+\left(2 d_{1}-d_{4}\right) f_{2}(x)+2 f_{3}(x) & =\varphi_{2}(x)-d_{4} \varphi_{1}(x) ; \\
\left(d_{1}^{3}-d_{1}^{2} d_{4}-\left(d_{1}^{2}-d_{1} d_{4}\right)\left(3 d_{1}-d_{4}\right)\right) f_{1}(x)+\left(3 d_{1}^{2}-2 d_{1} d_{4}-\left(2 d_{1}-d_{4}\right)\left(3 d_{1}-d_{4}\right)\right) f_{2}(x) & = \\
\varphi_{3}(x)-d_{4} \varphi_{2}(x)-\left(3 d_{1}-d_{4}\right)\left(\varphi_{2}(x)-d_{4} \varphi_{1}(x)\right), &
\end{aligned}
$$

instead of $f_{2}(x)$ by $f_{1}(x)$, we obtain third-order ODE for $f_{1}(x)$

$$
\begin{aligned}
& \left(3 d_{1}^{2}-2 d_{1} d_{4}-\left(2 d_{1}-d_{4}\right)\left(3 d_{1}-d_{4}\right)\right)\left(\varphi_{1}(x)-d_{4} \varphi_{0}(x)-\left(d_{1}-d_{4}\right) f_{1}(x)\right) \\
& \quad+\left(d_{1}^{3}-d_{1}^{2} d_{4}-\left(d_{1}^{2}-d_{1} d_{4}\right)\left(3 d_{1}-d_{4}\right)\right) f_{1}(x)=\varphi_{3}(x)-d_{4} \varphi_{2}(x)-\left(3 d_{1}-d_{4}\right)\left(\varphi_{2}(x)-d_{4} \varphi_{1}(x)\right)
\end{aligned}
$$

or

$$
\left(d_{1}-d_{4}\right)^{3} f_{1}(x)=\Phi(x)
$$


where

$$
\Phi(x)=\varphi_{3}(x)-3 d_{1} \varphi_{2}(x)+3 d_{1}^{2} \varphi_{1}(x)-d_{4}\left(3 d_{1}^{2}-3 d_{1} d_{4}+d_{4}^{2}\right) \varphi_{0}(x) .
$$

After solving (10), we have:

$$
f_{1}(x)=e^{\frac{b-b^{(4)}}{a-a^{(4)}}}\left(C_{1}+x C_{2}+x^{2} C_{3}\right)+\Psi(x),
$$

with $\Psi(x)=\frac{1}{2\left(a-a^{(4)}\right)^{3}} \int_{0}^{x} \Phi(z) e^{\frac{b-b^{(4)}}{a-a^{(4)}}(x-z)}(x-z)^{2} d z$,

where $C_{1}, C_{2}$ and $C_{3}$ are arbitrary integration constants. Then, using (11) and the first three equations of system of differential equations, it is easy to find three other functions for the solution of (9):

$$
\begin{aligned}
& f_{4}(x)=\varphi_{0}(x)-e^{\frac{b-b^{(4)}}{a-a^{(4)}} x}\left(C_{1}+x C_{2}+x^{2} C_{3}\right)-\Psi(x), \\
& f_{2}(x)=\varphi_{1}(x)-\left(d_{1}-d_{4}\right) \Psi(x)-d_{4} \varphi_{0}(x)-\left(a-a^{(4)}\right) e^{\frac{b-b^{(4)}}{a-a^{(4)}}\left(C_{2}+2 x C_{3}\right),} \\
& f_{3}(x)=\frac{1}{2}\left(\varphi_{2}(x)-d_{1}^{2} f_{1}(x)-2 d_{1} f_{2}(x)-d_{4}^{2} f_{4}(x)\right) \\
& =\frac{1}{2} \varphi_{2}(x)-\frac{1}{2}\left(d_{1}^{2}-d_{4}^{2}\right) \Psi(x)-\frac{1}{2} d_{4}^{2} \varphi_{0}(x)-d_{1} \varphi_{1}(x)+d_{1}\left(d_{1}-d_{4}\right) \Psi(x)+d_{1} d_{4} \varphi_{0}(x) \\
& +e^{\frac{b-b^{(4)}}{a-a^{(4)}} x}\left(\left(a^{(4)}\right)^{2} C_{3}-b a^{(4)} C_{2}-2 b a^{(4)} C_{3} x-a^{2} C_{3}+a b^{(4)} C_{2}+2 a b^{(4)} C_{3} x\right) \\
& +e^{\frac{b-b^{(4)}}{a-a^{(4)}} x}\left(-2 a a^{(4)} C_{3}+b a^{(4)} C_{2}+2 b a^{(4)} C_{3} x+2 a^{2} C_{3}-a b^{(4)} C_{2}-2 a b^{(4)} C_{3} x\right) .
\end{aligned}
$$

Now, substituting $f_{1}(x), f_{2}(x), f_{3}(x), f_{4}(x)$ into 9 , we get the solution:

$$
\begin{aligned}
u(t, x)=e^{-b t}\left(\Psi(x+a t)+t\left(\varphi_{1}(x+a t)-\left(d_{1}-d_{4}\right) \Psi(x+a t)-d_{4} \varphi_{0}(x+a t)\right)\right) & \\
& +e^{-b t} t^{2}\left(\frac{1}{2} \varphi_{2}(x+a t)-\frac{1}{2}\left(d_{1}^{2}-d_{4}^{2}\right) \Psi(x+a t)-\frac{1}{2} d_{4}^{2} \varphi_{0}(x+a t)\right) \\
+ & e^{-b t} t^{2}\left(-d_{1} \varphi_{1}(x+a t)+d_{1}\left(d_{1}-d_{4}\right) \Psi(x+a t)+d_{1} d_{4} \varphi_{0}(x+a t)\right) \\
& +e^{-b^{(4)} t}\left(\varphi_{0}\left(x+a^{(4)} t\right)-\Psi\left(x+a^{(4)} t\right)\right) .
\end{aligned}
$$

Case 3: We have coefficients of equation (5) satisfy $a^{(k)}=a, b^{(k)}=b$ with $k=1,2, a^{(k)}=c, b^{(k)}=d$ with $k=3,4, c \neq a$ and $b, d$ - arbitrary constants. According to equation (7), the general solution of equation (5) in this case has the form:

$$
u(t, x)=e^{-b t}\left(f_{1}(x+a t)+t f_{2}(x+a t)\right)+e^{-d t}\left(f_{3}(x+c t)+t f_{4}(x+c t)\right),
$$

we compute partial derivatives of first,second and third order in $\mathrm{t}$ and substitute them into the initial conditions (4), we get the following system of differential equations on the functions $f_{k}(x)$ with $k=1,2,3,4$ :

$$
\begin{array}{r}
f_{1}(x)+f_{3}(x)=\varphi_{0}(x) ; \\
-b f_{1}(x)+f_{2}(x)-d f_{3}(x)+f_{4}(x)+a f_{1}^{\prime}(x)+c f_{3}^{\prime}(x)=\varphi_{1}(x) ; \\
b^{2} f_{1}(x)+d^{2} f_{3}(x)-2 b f_{2}(x)-2 a b f_{1}^{\prime}(x)+2 a f_{2}^{\prime}(x)-2 d f_{4}(x)-2 c d f_{3}^{\prime}(x)+2 c f_{4}^{\prime}(x) \\
+a^{2} f_{1}^{\prime \prime}(x)+c^{2} f_{3}^{\prime \prime}(x)=\varphi_{2}(x) ; \\
-b^{3} f_{1}(x)-d^{3} f_{3}(x)+3 b^{2} f_{2}(x)+3 b^{2} a f_{1}^{\prime}(x)+3 d^{2} f_{4}(x)+3 d^{2} c f_{3}^{\prime}(x)-6 a b f_{2}^{\prime}(x) \\
3 b a^{2} f_{1}^{\prime \prime}(x)+3 a^{2} f_{2}^{\prime \prime}(x)-6 c d f_{4}^{\prime}(x)-3 d c^{2} f_{3}^{\prime \prime}(x)+3 c^{2} f_{4}^{\prime \prime}(x)+a^{3} f_{1}^{\prime \prime \prime}(x)+c^{3} f_{3}^{\prime \prime \prime}(x)=\varphi_{3}(x) .
\end{array}
$$

We introduce the following notation for differential operators: $d_{1}=a d / d x-b, d_{2}=c d / d x-d$ and $d_{1}^{j}=(a d / d x-b)^{j}, d_{2}^{j}=(c d / d x-d)^{j}$. In this notation, we rewrite system of differential equations for the 
unknown function $f_{1}(x), f_{2}(x), f_{3}(x), f_{4}(x)$ in the form:

$$
\begin{aligned}
f_{1}(x)+f_{3}(x) & =\varphi_{0}(x) ; \\
d_{1} f_{1}(x)+f_{2}(x)+d_{2} f_{3}(x)+f_{4}(x) & =\varphi_{1}(x) ; \\
d_{1}^{2} f_{1}(x)+2 d_{1} f_{2}(x)+d_{2}^{2} f_{3}(x)+2 d_{2} f_{4}(x) & =\varphi_{2}(x) ; \\
d_{1}^{3} f_{1}(x)+3 d_{1}^{2} f_{2}(x)+d_{2}^{3} f_{3}(x)+3 d_{2}^{2} f_{4}(x) & =\varphi_{3}(x),
\end{aligned}
$$

instead of $f_{2}(x), f_{3}(x), f_{4}(x)$ by $f_{1}(x)$, we obtain third-order ODE for $f_{1}(x)$ :

$$
\begin{aligned}
&\left(\frac{-1}{2} d_{1}^{3}+\frac{3}{2} d_{2} d_{1}^{2}+\frac{1}{2} d_{2}^{3}-\frac{3}{2} d_{1} d_{2}^{2}\right) f_{1}(x)= \\
& \varphi_{3}(x)-\frac{3}{2} d_{2} \varphi_{2}(x)-\frac{3}{2} d_{1} \varphi_{2}(x)+3 d_{1} d_{2} \varphi_{1}(x)+\frac{1}{2} d_{2}^{3} \varphi_{0}(x)-\frac{3}{2} d_{1} d_{2}^{2} \varphi_{0}(x),
\end{aligned}
$$

or

$$
\left(d_{1}^{3}-3 d_{2} d_{1}^{2}+3 d_{1} d_{2}^{2}-d_{2}^{3}\right) f_{1}(x)=\Phi(x),
$$

where $\Phi(x)=-2 \varphi_{3}(x)+3 d_{2} \varphi_{2}(x)+3 d_{1} \varphi_{2}(x)-6 d_{1} d_{2} \varphi_{1}(x)-d_{2}^{3} \varphi_{0}(x)+3 d_{1} d_{2}^{2} \varphi_{0}(x)$.

Solving the third-order differential equation from (13), we get:

$$
f_{1}(x)=e^{\frac{b-d}{a-c} x}\left(C_{1}+x C_{2}+x^{2} C_{3}\right)+\frac{1}{2(a-c)^{3}} \int_{0}^{x} \Phi(z) e^{\frac{b-d}{a-c}(x-z)}(x-z)^{2} d z,
$$

with $\Psi(x)=\frac{1}{2(a-c)^{3}} \int_{0}^{x} \Phi(z) e^{\frac{b-d}{a-c}(x-z)}(x-z)^{2} d z$, we obtain:

$$
f_{1}(x)=e^{\frac{b-d}{a-c} x}\left(C_{1}+x C_{2}+x^{2} C_{3}\right)+\Psi(x),
$$

where $C_{1}, C_{2}$ and $C_{3}$ are arbitrary integration constants. Then, using function 14 and the first three equations of system of differential equations it is easy to find three other functions for the solution of (12):

$$
\begin{aligned}
f_{3}(x) & =\varphi_{0}(x)-e^{\frac{b-d}{a-c} x}\left(C_{1}+x C_{2}+x^{2} C_{3}\right)-\Psi(x), \\
f_{2}(x) & =C_{4} e^{\frac{b-d}{a-c} x}-(a-c) C_{3} e^{\frac{b-d}{a-c} x} x \\
& +\int_{0}^{x} \frac{\left(\varphi_{2}(z)-2 d_{2} \varphi_{1}(z)-\left(d_{1}^{2}-2 d_{2} d_{1}\right) \Psi(z)+d_{2}^{2} \varphi_{0}(z)-d_{2}^{2} \Psi(z)\right) e^{\frac{b-d}{a-c}(x-z)}}{2(a-c)} d z, \\
f_{4}(x) & =\varphi_{1}(x)-d_{1} \Psi(x)-d_{2} \varphi_{0}(x)+d_{2} \Psi(x)-C_{4} e^{\frac{b-d}{a-c} x}+(a-c) C_{3} e^{\frac{b-d}{a-c} x} x \\
& -\int_{0}^{x} \frac{\left(\varphi_{2}(z)-2 d_{2} \varphi_{1}(z)-\left(d_{1}^{2}-2 d_{2} d_{1}\right) \Psi(z)+d_{2}^{2} \varphi_{0}(z)-d_{2}^{2} \Psi(z)\right) e^{\frac{b-d}{a-c}(x-z)}}{2(a-c)} d z \\
& -(a-c) e^{\frac{b-d}{a-c} x}\left(C_{2}+2 C_{3} x\right) .
\end{aligned}
$$

Now, substitute $f_{1}(x), f_{2}(x), f_{3}(x), f_{4}(x)$ into (12), we get the solution:

$$
\begin{aligned}
u(t, x)=e^{-b t} \Psi(x+a t) & +t e^{-b t} \Omega(x+a t)+e^{-d t} \varphi_{0}(x+c t)-e^{-d t} \Psi(x+c t) \\
& +t e^{-d t}\left(-\Omega(x+c t)+\varphi_{1}(x+c t)-d_{1} \Psi(x+c t)-d_{2} \varphi_{0}(x+c t)+d_{2} \Psi(x+c t)\right),
\end{aligned}
$$

where

$$
\Omega(x)=\int_{0}^{x} \frac{\left(\varphi_{2}(z)-2 d_{2} \varphi_{1}(z)-\left(d_{1}^{2}-2 d_{2} d_{1}\right) \Psi(z)+d_{2}^{2} \varphi_{0}(z)-d_{2}^{2} \Psi(z)\right) e^{\frac{b-d}{a-c}(x-z)}}{2(a-c)} d z .
$$


Case 4: We have coefficients of equation (5) satisfy $a^{(k)}=a, b^{(k)}=b$ with $k=1,2, a^{(3)}=c, b^{(3)}=d, a^{(4)}=e$, $b^{(4)}=f, c \neq a \neq e$ and $(b-d)(a-e) \neq(b-f)(a-c)$. According to equation (7), the general solution of equation (5) in this case has the form:

$$
u(t, x)=e^{-b t}\left(f_{1}(x+a t)+t f_{2}(x+a t)\right)+e^{-d t} f_{3}(x+c t)+e^{-f t} f_{4}(x+e t),
$$

we compute partial derivatives of first,second and third order in $t$ and substitute them into the initial conditions (4), we get the following system of differential equations on the functions $f_{k}(x)$ with $k=1,2,3,4$ :

$$
\begin{aligned}
f_{1}(x)+f_{3}(x)+f_{4}(x) & =\varphi_{0}(x) ; \\
-b f_{1}(x)+f_{2}(x)-d f_{3}(x)-f f_{4}(x)+a f_{1}^{\prime}(x)+c f_{3}^{\prime}(x)+e f_{4}^{\prime}(x) & =\varphi_{1}(x) ; \\
b^{2} f_{1}(x)+d^{2} f_{3}(x)+f^{2} f_{4}(x)-2 b f_{2}(x)-2 a b f_{1}^{\prime}(x)+2 a f_{2}^{\prime}(x) & \\
-2 c d f_{3}^{\prime}(x)-2 e f f_{4}^{\prime}(x)+a^{2} f_{1}^{\prime \prime}(x)+c^{2} f_{3}^{\prime \prime}(x)+e^{2} f_{4}^{\prime \prime}(x) & =\varphi_{2}(x) ; \\
-b^{3} f_{1}(x)-d^{3} f_{3}(x)-f^{3} f_{4}(x)+3 b^{2} f_{2}(x)+3 b^{2} a f_{1}^{\prime}(x)+3 c d^{2} f_{3}^{\prime}(x)+3 e f^{2} f_{4}^{\prime}(x)-6 a b f_{2}^{\prime}(x) & \\
-3 b a^{2} f_{1}^{\prime \prime}(x)+3 a^{2} f_{2}^{\prime \prime}(x)-3 c^{2} d f_{3}^{\prime \prime}(x)-3 e^{2} f f_{4}^{\prime \prime}(x)+a^{3} f_{1}^{\prime \prime \prime}(x)+c^{3} f_{3}^{\prime \prime \prime}(x)+e^{3} f_{4}^{\prime \prime \prime}(x) & =\varphi_{3}(x) .
\end{aligned}
$$

We introduce the following notation of differential operators: $d_{1}=a d / d x-b, d_{3}=c d / d x-d, d_{4}=e d / d x-f$ and $d_{1}^{j}=(a d / d x-b)^{j}, d_{3}^{j}=(c d / d x-d)^{j}, d_{4}^{j}=(e d / d x-f)^{j}$. In this notation, we rewrite system of differential equations for the unknown function $f_{1}(x), f_{2}(x), f_{3}(x), f_{4}(x)$ in the form:

$$
\begin{aligned}
f_{1}(x)+f_{3}(x)+f_{4}(x) & =\varphi_{0}(x), \\
d_{1} f_{1}(x)+f_{2}(x)+d_{3} f_{3}(x)+d_{4} f_{4}(x) & =\varphi_{1}(x), \\
d_{1}^{2} f_{1}(x)+2 d_{1} f_{2}(x)+d_{3}^{2} f_{3}(x)+d_{4}^{2} f_{4}(x) & =\varphi_{2}(x), \\
d_{1}^{3} f_{1}(x)+3 d_{1}^{2} f_{2}(x)+d_{3}^{3} f_{3}(x)+d_{4}^{3} f_{4}(x) & =\varphi_{3}(x),
\end{aligned}
$$

or

$$
\begin{aligned}
f_{1}(x)+f_{3}(x)+f_{4}(x) & =\varphi_{0}(x), \\
\left(d_{1}-d_{4}\right) f_{1}(x)+f_{2}(x)+\left(d_{3}-d_{4}\right) f_{3}(x) & =\varphi_{1}(x)-d_{4} \varphi_{0}(x), \\
\left(d_{1}^{2}-d_{4} d_{1}\right) f_{1}(x)+\left(2 d_{1}-d_{4}\right) f_{2}(x)+\left(d_{3}^{2}-d_{3} d_{4}\right) f_{3}(x) & =\varphi_{2}(x)-d_{4} \varphi_{1}(x), \\
\left(d_{1}^{3}-d_{4} d_{1}^{2}\right) f_{1}(x)+\left(3 d_{1}^{2}-2 d_{4} d_{1}\right) f_{2}(x)+\left(d_{3}^{3}-d_{4} d_{3}^{2}\right) f_{3}(x) & =\varphi_{3}(x)-d_{4} \varphi_{2}(x),
\end{aligned}
$$

or

$$
\begin{aligned}
f_{1}(x)+f_{3}(x)+f_{4}(x) & =\varphi_{0}(x), \\
\left(d_{1}-d_{4}\right) f_{1}(x)+f_{2}(x)+\left(d_{3}-d_{4}\right) f_{3}(x) & =\varphi_{1}(x)-d_{4} \varphi_{0}(x), \\
\left(d_{1}^{2}-d_{4} d_{1}-d_{3} d_{1}+d_{3} d_{4}\right) f_{1}(x)+\left(2 d_{1}-d_{4}-d_{3}\right) f_{2}(x) & =\varphi_{2}(x)-d_{4} \varphi_{1}(x)-d_{3} \varphi_{1}(x)+d_{3} d_{4} \varphi_{0}(x), \\
\left(d_{1}^{3}-d_{4} d_{1}^{2}-d_{3} d_{1}^{2}+d_{3} d_{4} d_{1}\right) f_{1}(x)+\left(3 d_{1}^{2}-2 d_{4} d_{1}-2 d_{3} d_{1}+d_{3} d_{4}\right) f_{2}(x) & =\varphi_{3}(x)-d_{4} \varphi_{2}(x)-d_{3} \varphi_{2}(x)+d_{3} d_{4} \varphi_{1}(x),
\end{aligned}
$$

instead of $f_{1}(x)$ by $f_{2}(x)$, we obtain second-order ODE for $f_{2}(x)$ :

$$
\begin{aligned}
& \left(d_{1}^{2}-d_{4} d_{1}-d_{3} d_{1}+d_{3} d_{4}\right) f_{2}(x)= \\
& \varphi_{3}(x)-d_{4} \varphi_{2}(x)-d_{3} \varphi_{2}(x)+d_{3} d_{4} \varphi_{1}(x)-d_{1} \varphi_{2}(x)+d_{1} d_{4} \varphi_{1}(x)+d_{1} d_{3} \varphi_{1}(x)-d_{1} d_{3} d_{4} \varphi_{0}(x),
\end{aligned}
$$

or

$$
\left(d_{1}^{2}-d_{4} d_{1}-d_{3} d_{1}+d_{3} d_{4}\right) f_{2}(x)=\Phi(x)
$$

where

$$
\Phi(x)=\varphi_{3}(x)-d_{4} \varphi_{2}(x)-d_{3} \varphi_{2}(x)+d_{3} d_{4} \varphi_{1}(x)-d_{1} \varphi_{2}(x)+d_{1} d_{4} \varphi_{1}(x)+d_{1} d_{3} \varphi_{1}(x)-d_{1} d_{3} d_{4} \varphi_{0}(x) .
$$

Solving the second-order differential equation from (16), we get:

$$
f_{2}(x)=C_{1} e^{\frac{b-d}{a-c} x}+C_{2} e^{\frac{b-f}{a-e} x}+\int_{0}^{x} \frac{\Phi(z)\left(e^{\frac{b-d}{a-c}(x-z)}-e^{\frac{b-f}{a-e}(x-z)}\right)}{b(c-e)+d(e-a)+f(a-c)} d z,
$$

with $\Omega(x)=\int_{0}^{x} \frac{\Phi(z)\left(e^{\frac{b-d}{a-c}(x-z)}-e^{\frac{b-f}{a-e}(x-z)}\right)}{b(c-e)+d(e-a)+f(a-c)} d z$, we obtain:

$$
f_{2}(x)=C_{1} e^{\frac{b-d}{a-c} x}+C_{2} e^{\frac{b-f}{a-e} x}+\Omega(x),
$$


Then, using function (17), we find three other functions for the solution of (15):

$$
\begin{aligned}
& f_{1}(x)=C_{3} e^{\frac{b-d}{a-c} x}+C_{4} e^{\frac{b-f}{a-e} x}-\int_{0}^{x} \frac{\left(2 d_{1}-d_{4}-d_{3}\right) \Omega(z)\left(e^{\frac{b-d}{a-c}(x-z)}-e^{\frac{b-f}{a-e}(x-z)}\right)}{b(c-e)+d(e-a)+f(a-c)} d z \\
&+\int_{0}^{x} \frac{\left(\varphi_{2}(z)-d_{4} \varphi_{1}(z)-d_{3} \varphi_{1}(z)+d_{3} d_{4} \varphi_{0}(z)\right)\left(e^{\frac{b-d}{a-c}(x-z)}-e^{\frac{b-f}{a-e}(x-z)}\right)}{b(c-e)+d(e-a)+f(a-c)} d z \\
&-\int_{0}^{x} \frac{\left((a-e) C_{1} e^{\frac{b-d}{a-c} z}-(a-c) C_{2} e^{\frac{b-f}{a-e} z}\right)\left(e^{\frac{b-d}{a-c}(x-z)}-e^{\frac{b-f}{a-e}(x-z)}\right)}{(a-c)(a-e)} d z \\
&=\Theta(x)+C_{3} e^{\frac{b-d}{a-c} x}+C_{4} e^{\frac{b-f}{a-e} x}-\int_{0}^{x} \frac{\left((a-e) C_{1} e^{\frac{b-d}{a-c} z}-(a-c) C_{2} e^{\frac{b-f}{a-e} z}\right)\left(e^{\frac{b-d}{a-c}(x-z)}-e^{\frac{b-f}{a-e}(x-z)}\right)}{(a-c)(a-e)} d z
\end{aligned}
$$

with

$$
\begin{gathered}
\Theta(x)=-\int_{0}^{x} \frac{\left(2 d_{1}-d_{4}-d_{3}\right) \Omega(z)\left(e^{\frac{b-d}{a-c}(x-z)}-e^{\frac{b-f}{a-e}(x-z)}\right)}{b(c-e)+d(e-a)+f(a-c)} d z \\
+\int_{0}^{x} \frac{\left(\varphi_{2}(z)-d_{4} \varphi_{1}(z)-d_{3} \varphi_{1}(z)+d_{3} d_{4} \varphi_{0}(z)\right)\left(e^{\frac{b-d}{a-c}(x-z)}-e^{\frac{b-f}{a-e}(x-z)}\right)}{b(c-e)+d(e-a)+f(a-c)} d z,
\end{gathered}
$$

and

$$
\begin{aligned}
& f_{3}(x)=C_{5} e^{\frac{d-f}{c-e} x}+\int_{0}^{x} \frac{\left(\varphi_{1}(z)-d_{4} \varphi_{0}(z)-\left(d_{1}-d_{4}\right) f_{1}(z)-f_{2}(z)\right) e^{\frac{d-f}{c-e}(x-z)}}{(c-e)} d z \\
& =C_{5} e^{\frac{d-f}{c-e} x}+\int_{0}^{x} \frac{\left(\varphi_{1}(z)-d_{4} \varphi_{0}(z)-\left(d_{1}-d_{4}\right) \Theta(z)-\Omega(z)\right) e^{\frac{d-f}{c-e}(x-z)}}{(c-e)} d z \\
& -\int_{0}^{x} \frac{\left(C_{1} e^{\frac{b-d}{a-c} z}+C_{2} e^{\frac{b-f}{a-e} z}-(b-f)\left(C_{3} e^{\frac{b-d}{a-c} z}+C_{4} e^{\frac{b-f}{a-e} z}\right) e^{\frac{d-f}{c-e}(x-z)}\right.}{(c-e)} d z \\
& -\int_{0}^{x} \int_{0}^{z} \frac{(b-f)\left((a-e) C_{1} e^{\frac{b-d}{a-c} t}-(a-c) C_{2} e^{\frac{b-f}{a-e} t}\right)\left(e^{\frac{b-d}{a-c}(z-t)}-e^{\frac{b-f}{a-e}(z-t)}\right) e^{\frac{d-f}{c-e}(x-z)}}{(a-c)(a-e)(c-e)} d t d z \\
& -\int_{0}^{x} \frac{\left((a-e)\left(\frac{b-d}{a-c} C_{3} e^{\frac{b-d}{a-c} z}+\frac{b-f}{a-e} C_{4} e^{\frac{b-f}{a-e} z}\right)\right) e^{\frac{d-f}{c-e}(x-z)}}{(c-e)} d z \\
& +\int_{0}^{x} \int_{0}^{z} \frac{\left((a-e) C_{1} e^{\frac{b-d}{a-c} t}-(a-c) C_{2} e^{\frac{b-f}{a-e} t}\right)\left(\frac{b-d}{a-c} e^{\frac{b-d}{a-c}(z-t)}-\frac{b-f}{a-e} e^{\frac{b-f}{a-e}(z-t)}\right) e^{\frac{d-f}{c-e}(x-z)}}{(a-c)(c-e)} d t d z,
\end{aligned}
$$




$$
\begin{aligned}
& f_{4}(x)= \varphi_{0}(x)-\Theta(x)-\int_{0}^{x} \frac{\left(\varphi_{1}(z)-d_{4} \varphi_{0}(z)-\left(d_{1}-d_{4}\right) \Theta(z)-\Omega(z)\right) e^{\frac{d-f}{c-e}(x-z)}}{(c-e)} d z \\
&+\int_{0}^{x} \frac{\left((a-e) C_{1} e^{\frac{b-d}{a-c} z}-(a-c) C_{2} e^{\frac{b-f}{a-e} z}\right)\left(e^{\frac{b-d}{a-c}(x-z)}-e^{\frac{b-f}{a-e}(x-z)}\right)}{(a-c)(a-e)} d z \\
&+\int_{0}^{x} \frac{\left(C_{1} e^{\frac{b-d}{a-c} z}+C_{2} e^{\frac{b-f}{a-e} z}-(b-f)\left(C_{3} e^{\frac{b-d}{a-c} z}+C_{4} e^{\frac{b-f}{a-e} z}\right) e^{\frac{d-f}{c-e}(x-z)}\right.}{(c-e)} d z \\
&+ \int_{0}^{x} \int_{0}^{z} \frac{(b-f)\left((a-e) C_{1} e^{\frac{b-d}{a-c} t}-(a-c) C_{2} e^{\frac{b-f}{a-e} t}\right)\left(e^{\frac{b-d}{a-c}(z-t)}-e^{\frac{b-f}{a-e}(z-t)}\right) e^{\frac{d-f}{c-e}(x-z)}}{(a-e)(c-e)} d t d z \\
&+\int_{0}^{x} \frac{\left((a-e)\left(\frac{b-d}{a-c} C_{3} e^{\frac{b-d}{a-c} z}+\frac{b-f}{a-e} C_{4} e^{\frac{b-f}{a-e} z}\right)\right) e^{\frac{d-f}{c-e}(x-z)}}{(c-e)} d z \\
& \int_{0}^{x} \frac{\left.(a-e) C_{1} e^{\frac{b-d}{a-c} t}-(a-c) C_{2} e^{\frac{b-f}{a-e} t}\right)\left(\frac{b-d}{a-c} e^{\frac{b-d}{a-c}(z-t)}-\frac{b-f}{a-e} e^{\frac{b-f}{a-e}(z-t)}\right) e^{\frac{d-f}{c-e}(x-z)}}{(a-c)(c-e)} d t d z \\
&-C_{3} e^{\frac{b-d}{a-c} x}-C_{4} e^{\frac{b-f}{a-e} x}-C_{5} e^{\frac{d-f}{c-e} x} .
\end{aligned}
$$

After substitution of $f_{1}(x), f_{2}(x), f_{3}(x), f_{4}(x)$ into equation (15), we get a solution of the Cauchy problem in this case :

$$
\begin{array}{r}
u(t, x)=e^{-b t} \Theta(x+a t)+t e^{-b t} \Omega(x+a t)+e^{-f t} \varphi_{0}(x+e t)-e^{-f t} \Theta(x+e t) \\
+e^{-d t} \int_{0}^{x+c t} \frac{\left(\varphi_{1}(z)-d_{4} \varphi_{0}(z)-\left(d_{1}-d_{4}\right) \Theta(z)-\Omega(z)\right) e^{\frac{d-f}{c-e}(x+c t-z)}}{(c-e)} d z \\
-e^{-f t} \int_{0}^{x+e t} \frac{\left(\varphi_{1}(z)-d_{4} \varphi_{0}(z)-\left(d_{1}-d_{4}\right) \Theta(z)-\Omega(z)\right) e^{\frac{d-f}{c-e}(x+e t-z)}}{(c-e)} d z
\end{array}
$$

Case 5: We have coefficients of equation (5) satisfy $a^{(i)} \neq a^{(j)}$ with $\forall i, j=\overline{1,4}$ and $\left(b^{(1)}-b^{(2)}\right)\left(a^{(1)}-a^{(3)}\right) \neq$ $\left(b^{(1)}-b^{(3)}\right)\left(a^{(1)}-a^{(2)}\right),\left(b^{(1)}-b^{(2)}\right)\left(a^{(1)}-a^{(4)}\right) \neq\left(b^{(1)}-b^{(4)}\right)\left(a^{(1)}-a^{(2)}\right)$. According to equation (7), the general solution of equation (5) in this case has the form:

$$
u(t, x)=e^{-b^{(1)} t} f_{1}\left(x+a^{(1)} t\right)+e^{-b^{(2)} t} f_{2}\left(x+a^{(2)} t\right)+e^{-b^{(3)} t} f_{3}\left(x+a^{(3)} t\right)+e^{-b^{(4)} t} f_{4}\left(x+a^{(4)} t\right),
$$

we compute partial derivatives of first,second and third order in $t$ and substitute them into the initial conditions (4), we get the following system of differential equations on the functions $f_{k}(x)$ with $k=1,2,3,4$ and we introduce the following notation for differential operators: $d_{i}=a^{(i)} d / d x-b^{(i)}$, and $d_{i}^{j}=\left(a^{(i)} d / d x-b^{(i)}\right)$ with $i=\overline{1,4}$ and $j=\overline{1,3}$. In this notation, we rewrite the system of differential equations for the unknown function $f_{1}(x)$, $f_{2}(x), f_{3}(x), f_{4}(x)$ in the form:

$$
\begin{aligned}
f_{1}(x)+f_{2}(x)+f_{3}(x)+f_{4}(x) & =\varphi_{0}(x) ; \\
d_{1} f_{1}(x)+d_{2} f_{2}(x)+d_{3} f_{3}(x)+d_{4} f_{4}(x) & =\varphi_{1}(x) ; \\
d_{1}^{2} f_{1}(x)+d_{2}^{2} f_{2}(x)+d_{3}^{2} f_{3}(x)+d_{4}^{2} f_{4}(x) & =\varphi_{2}(x) ; \\
d_{1}^{3} f_{1}(x)+d_{2}^{3} f_{2}(x)+d_{3}^{3} f_{3}(x)+d_{4}^{3} f_{4}(x) & =\varphi_{3}(x) ;
\end{aligned}
$$

or

$$
\begin{aligned}
f_{1}(x)+f_{2}(x)+f_{3}(x)+f_{4}(x) & =\varphi_{0}(x) \\
\left(d_{1}-d_{4}\right) f_{1}(x)+\left(d_{2}-d_{4}\right) f_{2}(x)+\left(d_{3}-d_{4}\right) f_{3}(x) & =\varphi_{1}(x)-d_{4} \varphi_{0}(x) \\
\left(d_{1}^{2}-d_{1} d_{4}-d_{3} d_{1}+d_{3} d_{4}\right) f_{1}(x)+\left(d_{2}^{2}-d_{2} d_{4}-d_{3} d_{2}+d_{3} d_{4}\right) f_{2}(x) & =\varphi_{2}(x)-d_{4} \varphi_{1}(x)-d_{3} \varphi_{1}(x)+d_{3} d_{4} \varphi_{0}(x)
\end{aligned}
$$


instead of $f_{2}(x)$ by $f_{1}(x)$, we obtain third-order ODE for $f_{1}(x)$

$$
\begin{gathered}
\left(d_{1}^{3}-d_{4} d_{1}^{2}-d_{3} d_{1}^{2}+d_{3} d_{1} d_{4}\right) f_{1}(x)+\left(d_{2}^{3}-d_{4} d_{2}^{3}-d_{3} d_{2}^{2}+d_{3} d_{2} d_{4}\right) f_{2}(x)= \\
\varphi_{3}(x)-d_{4} \varphi_{2}(x)-d_{3} \varphi_{2}(x)+d_{3} d_{4} \varphi_{1}(x) ; \\
\left(d_{1}^{3}-d_{4} d_{1}^{2}-d_{3} d_{1}^{2}+d_{3} d_{1} d_{4}-d_{2} d_{1}^{2}+d_{2} d_{1} d_{4}+d_{2} d_{3} d_{1}-d_{2} d_{3} d_{4}\right) f_{1}(x)= \\
\varphi_{3}(x)-d_{4} \varphi_{2}(x)-d_{3} \varphi_{2}(x)+d_{3} d_{4} \varphi_{1}(x)-d_{2} \varphi_{2}(x)+d_{2} d_{4} \varphi_{1}(x)+d_{2} d_{3} \varphi_{1}(x)-d_{2} d_{3} d_{4} \varphi_{0}(x) ;
\end{gathered}
$$

where

$$
\Phi(x)=\varphi_{3}(x)-d_{4} \varphi_{2}(x)-d_{3} \varphi_{2}(x)+d_{3} d_{4} \varphi_{1}(x)-d_{2} \varphi_{2}(x)+d_{2} d_{4} \varphi_{1}(x)+d_{2} d_{3} \varphi_{1}(x)-d_{2} d_{3} d_{4} \varphi_{0}(x) .
$$

After solving this equation, we receive

$$
\begin{gathered}
f_{1}(x)=C_{1} e^{\frac{b^{(1)}-b^{(2)}}{a^{(1)}-a^{(2)}} x}+C_{2} e^{\frac{b^{(1)}-b^{(3)}}{a^{(1)}-a^{(3)} x}+C_{3} e^{\frac{b^{(1)}-b^{(4)}}{a^{(1)}-a^{(4)}} x}} \\
+\sum_{k=2}^{4} \int_{0}^{x} \frac{\Phi(z) e^{\frac{b^{(1)}-b^{(k)}}{a^{(1)}-a^{(k)}(x-z)}}}{P^{\prime}\left(\frac{b^{(1)}-b^{(k)}}{a^{(1)}-a^{(k)}}\right)\left(a^{(1)}-a^{(2)}\right)\left(a^{(1)}-a^{(3)}\right)\left(a^{(1)}-a^{(4)}\right)} d z \\
\quad=C_{1} e^{\frac{b^{(1)}-b^{(2)}}{a^{(1)}-a^{(2)}}+C_{2} e^{\frac{b^{(1)}-b^{(3)}}{a^{(1)}-a^{(3)}}}+C_{3} e^{\frac{b^{(1)}-b^{(4)}}{a^{(1)}-a^{(4)}}}+\Omega(x),}
\end{gathered}
$$

where $P(\lambda)=\left(\lambda-\frac{b^{(1)}-b^{(2)}}{a^{(1)}-a^{(2)}}\right)\left(\lambda-\frac{b^{(1)}-b^{(3)}}{a^{(1)}-a^{(3)}}\right)\left(\lambda-\frac{b^{(1)}-b^{(4)}}{a^{(1)}-a^{(4)}}\right)$ and

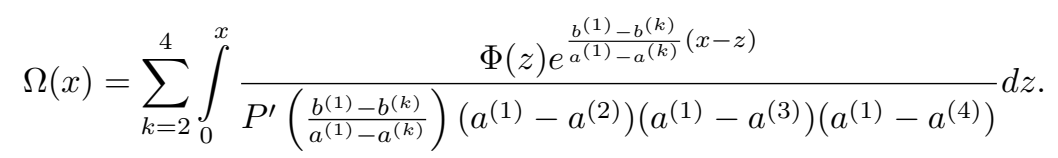

Then, using function $f_{1}(x)$, we find three other functions for the solution of (18):

$$
\begin{aligned}
& f_{2}(x)=\Psi(x)+C_{4} e^{\frac{b^{(2)}-b^{(3)}}{a^{(2)}-a^{(3)}}+}+C_{5}{\frac{b^{(2)}-b^{(4)}}{a^{(2)}-a^{(4)}}}^{x} \\
&-\sum_{k=2}^{4} \int_{0}^{x} \frac{\left(a^{(1)}-a^{(3)}\right)\left(a^{(1)}-a^{(4)}\right) C_{k-1}\left(b^{(1)}-b^{(k)}\right)^{2} \Pi(x, z)}{\left(a^{(1)}-a^{(k)}\right)^{2}\left(a^{(4)}\left(b^{(2)}-b^{(3)}\right)+a^{(2)}\left(b^{(3)}-b^{(4)}\right)+a^{(3)}\left(b^{(4)}-b^{(2)}\right)\right)} d z \\
&+\sum_{k=2}^{4} \int_{0}^{x} \frac{\left(b^{(1)}-b^{(3)}\right)\left(a^{(1)}-a^{(4)}\right) C_{k-1}\left(b^{(1)}-b^{(k)}\right) \Pi(x, z)}{\left(a^{(1)}-a^{(k)}\right)\left(a^{(4)}\left(b^{(2)}-b^{(3)}\right)+a^{(2)}\left(b^{(3)}-b^{(4)}\right)+a^{(3)}\left(b^{(4)}-b^{(2)}\right)\right)} d z \\
&+\sum_{k=2}^{4} \int_{0}^{x} \frac{\left(b^{(1)}-b^{(4)}\right)\left(a^{(1)}-a^{(3)}\right) C_{k-1}\left(b^{(1)}-b^{(k)}\right) \Pi(x, z)}{\left(a^{(1)}-a^{(k)}\right)\left(a^{(4)}\left(b^{(2)}-b^{(3)}\right)+a^{(2)}\left(b^{(3)}-b^{(4)}\right)+a^{(3)}\left(b^{(4)}-b^{(2)}\right)\right)} d z \\
& f_{3}(x)=-\sum_{0}^{x} \frac{\left.\left(\varphi_{1}(z)-d_{4} \int_{0}(z)-\left(d_{1}-d_{4}\right) f_{1}(z)-\left(d_{2}-d_{4}\right) f_{2}(z)\right) e^{\frac{b^{(3)}-b^{(4)}}{a^{(3)}(1)}(x-z)}-b^{(4)}\right)\left(b^{(1)}-b^{(3)}\right) C_{k-1} \Pi(x, z)}{a^{(3)}-a^{(4)}} d z+C_{6} e^{\frac{b^{(3)}-b^{(4)}}{a^{(3)}} x}, \\
& f_{4}(x)=\varphi_{0}(x)-f_{0}(x)-f_{1}(x)-f_{2}(x)-f_{3}(x),
\end{aligned}
$$

where

$$
\begin{aligned}
\Psi(x)=- & \int_{0}^{x} \frac{\left(\left(d_{1}^{2}-d_{1} d_{4}-d_{3} d_{1}+d_{3} d_{4}\right) \Omega(z)\right)\left(e^{\frac{b^{(2)}-b^{(4)}}{a^{(2)}-a^{(4)}}(x-z)}-e^{\left.\frac{b^{(2)}-b^{(3)}}{a^{(2)}-a^{(3)}(x-z)}\right)}\right.}{a^{(4)}\left(b^{(2)}-b^{(3)}\right)+a^{(2)}\left(b^{(3)}-b^{(4)}\right)+a^{(3)}\left(b^{(4)}-b^{(2)}\right)} d z \\
& +\int_{0}^{x} \frac{\left(\varphi_{2}(z)-d_{4} \varphi_{1}(z)-d_{3} \varphi_{1}(z)+d_{3} d_{4} \varphi_{0}(z)\right)\left(e^{\frac{b^{(2)}-b^{(4)}}{a^{(2)}-a^{(4)}}(x-z)}-e^{\frac{b^{(2)}-b^{(3)}}{a^{(2)}-a^{(3)}}(x-z)}\right)}{a^{(4)}\left(b^{(2)}-b^{(3)}\right)+a^{(2)}\left(b^{(3)}-b^{(4)}\right)+a^{(3)}\left(b^{(4)}-b^{(2)}\right)} d z,
\end{aligned}
$$




$$
\Pi(x, z)=e^{\frac{b^{(1)}-b^{(k)}}{a^{(1)}-a^{(k)}} z}\left(e^{\frac{b^{(2)}-b^{(4)}}{a^{(2)}-a^{(4)}}(x-z)}-e^{\frac{b^{(2)}-b^{(3)}}{a^{(2)}-a^{(3)}}(x-z)}\right) .
$$

After substitution of $f_{1}(x), f_{2}(x), f_{3}(x), f_{4}(x)$ into equation (18), we get a solution of the Cauchy problem in this case:

$$
\begin{gathered}
u(t, x)=e^{-b^{(1)} t} \Omega\left(x+a^{(1)} t\right)+e^{-b^{(2)} t} \Psi\left(x+a^{(2)} t\right) \\
+e^{-b^{(3)} t} \int_{0}^{x+a^{(3)} t} \frac{\left(\varphi_{1}(z)-d_{4} \varphi_{0}(z)\right) e^{\frac{b^{(3)}-b^{(4)}}{a^{(3)}}\left(x+a^{(3)} t-z\right)}}{a^{(3)}-a^{(4)}} d z \\
-e^{-b^{(3)} t} \int_{0}^{x+a^{(3)} t} \frac{\left(\left(d_{1}-d_{4}\right) \Omega(z)+\left(d_{2}-d_{4}\right) \Psi(z)\right) e^{\frac{b^{(3)}-b^{(4)}}{a^{(3)}-a^{(4)}}\left(x+a^{(3)} t-z\right)}}{a^{(3)}-a^{(4)}} d z \\
+e^{-b^{(4)} t}\left(\varphi_{0}\left(x+a^{(4)} t\right)-\Omega\left(x+a^{(4)} t\right)-\Psi\left(x+a^{(4)} t\right)\right) \\
-e^{-b^{(4)} t} \int_{0}^{x+a^{(4)} t} \frac{\left(\varphi_{1}(z)-d_{4} \varphi_{0}(z)\right) e^{\frac{b^{(3)}-b^{(4)}}{a^{(3)}}\left(x+a^{(4)} t-z\right)}}{a^{(3)}-a^{(4)}} d z \\
+e^{-b^{(4)} t} \int_{0}^{x+a^{(4)} t} \frac{\left(\left(d_{1}-d_{4}\right) \Omega(z)+\left(d_{2}-d_{4}\right) \Psi(z)\right) e^{\frac{b^{(3)}-b^{(4)}}{a^{(3)}-a^{(4)}}\left(x+a^{(4)} t-z\right)}}{a^{(3)}-a^{(4)}} d z .
\end{gathered}
$$

We obtain the following theorem:

Theorem 2.1. The Cauchy problem (4) - (5) has a unique classical solution in $C^{4}(\bar{Q})$ for arbitrary functions $\varphi_{j}(j=\overline{0,3})$ in the class $C^{3-j}(\mathbb{R}), j=\overline{0,3}$.

Now, consider the Cauchy problem for the inhomogeneous equation. Since the considered problem is linear, it follows that its solution $u$ can be represented as the sum of two functions $u=\bar{u}+v$, where $\bar{u}$ is a solution of problem (4) $-(5)$, and $v$ is a solution of following equations:

$$
\mathcal{L}^{(4)} v(t, x)=f(t, x), \quad(t, x) \in Q,
$$

with the homogeneous Cauchy conditions:

$$
\left.v\right|_{t=0}=0,\left.\quad \frac{\partial v}{\partial t}\right|_{t=0}=0,\left.\quad \frac{\partial^{2} v}{\partial t^{2}}\right|_{t=0}=0,\left.\quad \frac{\partial^{3} v}{\partial t^{3}}\right|_{t=0}=0 .
$$

We define the function $v(t, x)$ via the function $w(t, \tau, x)$ with a parameter $\tau \in[0, \infty)$ by the relation:

$$
v(t, x)=\int_{0}^{t} \omega(t-\tau, \tau, x) d \tau
$$

The function $w$ treated as a function of the independent variables $t$ and $x$ is a solution of the homogeneous equation (5) with the Cauchy conditions:

Indeed, we have:

$$
\left.\omega\right|_{t=\tau}=0,\left.\quad \frac{\partial \omega}{\partial t}\right|_{t=\tau}=0,\left.\quad \frac{\partial^{2} \omega}{\partial t^{2}}\right|_{t=\tau}=0,\left.\quad \frac{\partial^{3} \omega}{\partial t^{3}}\right|_{t=\tau}=f(\tau, x) .
$$

$$
\begin{gathered}
\left.v\right|_{t=0}=\int_{0}^{0} \omega(-\tau, \tau, x) d \tau=0, \\
\left.\frac{\partial v}{\partial t}\right|_{t=0}=\omega(0,0, x)+\int_{0}^{0} \frac{\partial \omega(t-\tau, \tau, x)}{\partial t} d \tau=0, \\
\left.\frac{\partial^{2} v}{\partial t^{2}}\right|_{t=0}=\frac{\partial \omega(0,0, x)}{\partial t}+\int_{0}^{0} \frac{\partial^{2} \omega(t-\tau, \tau, x)}{\partial t^{2}} d \tau=0,
\end{gathered}
$$




$$
\begin{gathered}
\left.\frac{\partial^{3} v}{\partial t^{3}}\right|_{t=0}=\frac{\partial^{2} \omega(0,0, x)}{\partial t^{2}}+\int_{0}^{0} \frac{\partial^{3} \omega(t-\tau, \tau, x)}{\partial t^{3}} d \tau=0 \\
\mathcal{L}^{(4)} v(t, x)=\prod_{k=1}^{4}\left(\partial_{t}-a^{(k)} \partial_{x}+b^{(k)}\right) v(t, x)=f(t, x)+\int_{0}^{t}\left(\mathcal{L}^{(4)} \omega(t-\tau, \tau, x)\right) d \tau=f(t, x) .
\end{gathered}
$$

Theorem 2.2. If the right-hand side of Eq. (3) belongs to the set $C^{0,4}(\bar{Q})$ and the functions $\varphi_{j}(j=\overline{0,3})$ occurring in condition (4) belong to the class $C^{3-j}(\mathbb{R})$, then for such arbitrary functions, there exists a unique classical solution $u=\bar{u}+v$ of problem (3), (4) in the class $C^{4}(Q)$, where $\bar{u}$ is the classical solution for problem (4), (5) and $v$ is the solution for problem (19), (20).

\section{References}

[1] Korzyuk V.I., Kozlovskaya I.S. Solution of the Cauchy Problem for a Hyperbolic Equation with Constant Coefficients in the Case of Two Independent Variables. Differential Equations, 2012, 48 (5), P. 700-709.

[2] Korzyuk V.I., Kozlovskaya I.S. Solution of the Cauchy Problem for a Hyperbolic Equation for a Homogeneous Differential Operator in the Case of Two Independent Variables. Dokl. NAN Belarusi, 2011, 55 (5), P. 9-13.

[3] Petrovskii I.G. On the Cauchy Problem for Systems of Linear Partial Differential Equations in a Domain of Non analytic Functions. Bull. Moskov. Univ. Mat. Mekh., 1938, 1 (7), P. 1-72.

[4] Korzyuk V.I. Equations of Mathematical Physics: lectures. Volume 4. Minsk, 2008 [in Russian].

[5] Korzyuk V.I., Kozlovskaya I.S. Cauchy Problem for a Hyperbolic Equation with Constant Coefficients in the Case of Two Independent Variables. Mathematical Modeling and Differential Equations: Proc. 3rd Int. Sci. Conf., September 17?-22, 2012, Brest-?Minsk, 2012, P. $171-176$.

[6] Korzyuk V.I., Kozlouskaya I.S., Kozlov A.I. Caushy Problem in Half-Plane for Hyperbolic Equation with Constant Coefficients. In Analytic Methods of Analysis and Differential Equations (AMADE 2012), Cambridge, 2013, P. 45-71.

[7] Gopalakrishnan S., Narendar S. Wave Propagation in Nanostructures. Springer International Publishing Switzerland, 2013.

[8] Jalilir N. Piezoelectric-Based Vibration Control-From Macro to Micro/Nano Scale Systems. Springer Science + Business Media, LLC, 2010.

[9] Doyle J.F. Wave propagation in structures. Springer, New York, 1999.

[10] Gopalakrishnan S., Mitra M. Wavelet Methods for Dynamical Problems With Application to Metallic, Composite, and Nano-Composite Structures. Taylor and Francis Group, LLC, 2010. 